\title{
Suicide Risk Assessment Must be Collaborative
}

\section{Konrad Michel*}

Professor of Psychiatry, University of Bern, Bern, Switzerland

*Corresponding Author: Konrad Michel, Professor of Psychiatry, University of

Bern, Bern, Switzerland.
Received: November 06, 2020

Published: November 18, 2020

(C) All rights are reserved by Konrad Michel.

\begin{abstract}
When faced with a patient who may have suicide ideas or plans, clinicians usually perform a suicide risk assessment. However, short-term suicide prediction is a virtually impossible task. Suicidal individuals often deny suicidal plans, even when the issue is addressed directly by the clinician. An important aspect of the communication problem between patient and clinician is that the traditional medical model of suicide does not match with the inner experience of the suicidal patient. Here, it is argued that suicide risk assessment must be a collaborative process, in which patient and clinician together explore the individual's actual suicide risk and jointly develop adequate measures to keep the patient safe.
\end{abstract}

Keywords: Suicide; Risk Assessment; Narrative

\section{Introduction}

For most medical professionals, suicide and suicide risk assessment is terra incognita. In the medical training physicians learn that acute suicide risk requires admission to a psychiatric institution, often against the patient's will. In the traditional medical model, suicidal behaviour is understood as a consequence of an underlying mental disorder, which itself is the expression of some disorder of brain function. Like in a somatic emergency situation, it is considered to be the clinician's responsibility to decide about the procedure to keep the patient safe. However, many patients keep suicidal thoughts and plans to themselves [1-3]. Suicide risk assessment is invariably related to the liability factor and the fear of litigation, should the patient die by suicide. In order to be on the safe side, clinicians tend to admit patients. In cases of involuntary hospitalization this may involve calling the police, who will take the handcuffed patient and admit to a psychiatric institution. However, psychiatric hospitals are not at all safe places, with high suicide rates during in-patient care and after discharge [4,5]. Many patients admitted because of suicide risk, in retrospect view hospitalization, particularly when involuntary, as a negative, if not a harmful experience [6].
No wonder the average medical person is in a state of alarm when faced with this situation. The suicidal patient is a mystery and a threat. Patients feel the uneasiness of the clinician, and, not surprisingly, patients are holding back with disclosing their inner experience of shame, pain, hopelessness, loss of self-esteem, and the wish to end it all.

\section{Risk assessment}

A huge number of suicide risk scales have been developed. Very few of them are used in clinical practice. The problem is that risk scales can help to identify patients with a long-term risk, but are of no help in predicting immediate and short-term risk. Risk scales are based on suicide risk factors. The main long-term risk factors have been known for long: A history of past suicide attempts is the strongest indicator of a long-term risk. After attempted suicide, the suicide risk is, probably life-long, increased 40 -fold or more [7,8], it is higher when the suicide attempt was medically serious [9], and it increases with further suicide attempts [10]. Other long-term risk factors are psychiatric disorders, such as affective disorders (depression, bipolar disorder), substance abuse, and personality disorders. This is an information that clinicians can normally get 
from a usual clinical interview. More recent scales like the Columbia-Suicide Severity Rating Scale [11] use a set of questions about suicidal thoughts, plans and choice of suicide method - based on the assumption that higher seriousness of intent goes along with higher suicide risk. Yet, an Australian team [12] found that even sophisticated suicide risk scales hardly add much to the usual clinical assessment. Carter and colleagues [13], from a systematic review and meta-analysis of the predictive value of risk scales, concluded that "no instrument is sufficiently accurate as a basis to determine allocation to intervention". Chan., et al. [14], in a review of risk factors and risk scales concluded that the scales and tools reviewed had poor predictive value. "The use of these scales or an over-reliance on the identification of risk factors in clinical practice, is, in our view, potentially dangerous and may provide false reassurance for clinicians and managers. The idea of risk assessment as risk prediction is a fallacy and should be recognised as such".

Busch., et al. [3] looked at the clinical charts of 76 patients who died by suicide while in hospital or immediately after discharge. Suicide risk had been rated by standard risk predictors. Seventyeight percent had denied suicidal ideation, and $28 \%$ had a no-suicide contract. In a Finnish study [1] eighteen percent of those who had contacted a physician had done so on the day of their suicide, yet even then the issue of suicide was addressed in only one fifth of these cases. Similar findings have been reported from Australia [15]. Obviously, a major problem is that suicidal persons, even when are under medical care, rarely talk about their intentions, nor do they spontaneously mention past suicide crises $[2,16]$.

\section{The Tower of Babel syndrome}

In order to find a solution to the problem of risk assessment we need to look at the communication between patient and clinician. Medical training equips health professionals with the skills to detect pathology and to diagnose somatic and psychiatric disorders, but it does not usually provide helpful models to understand the very personal psychological experience of the suicidal individual. Suicidal patients have repeatedly reported feeling ignored by health professionals, and to experience mental health care as unhelpful [17]. Interestingly, some of them said that nurses and social workers had been more helpful than doctors [18].

The problem with the medical conceptualization of suicidal behaviour is that it is based on a "linear model", that is, similar to somatic illness models, it assumes a causal pathology and a chain of biological and functional changes leading to specific symptoms, and finally to an illness-specific treatment. The association between mental illness and suicidal behaviour is based on the classical retrospective studies which found the typical symptoms a psychiatric diagnosis in over $90 \%$ of suicides $[19,20]$. This model of suicidal behaviour, however, mistakes risk factors for causal factors. Although psychiatric diagnoses are undoubtedly important risk factors for suicide [21], the illness-based model has a very limited potential to reduce suicidal behaviour on a population level $[22,23]$. Despite new developments in the pharmacotherapy of psychiatric disorders, there has been no general downwards trend in suicide rates over the last decades [24]. In the traditional medical approach, the suicidal person is a passive entity, driven by a psychiatric disorder, not a person with an individual biography, with her or his own inner world, with an emotional life, with life goals, and vulnerabilities.

The personal experience of suicidal patients is characterized by psychological pain, hopelessness and feelings of shame, a sense of personal failure, of being useless, a burden to others, and they hate themselves for this $[25,26]$. There is a serious communication problem: The patients' concepts of suicide and the concepts of professional helpers do not match. The two protagonists do not understand each other, they speak different languages - a true Tower of Babel syndrome. Disorders of mental health are risk factors for suicide, but they are not the cause. It is not the depression that kills, but the person who acts according to a personal logic.

The most obvious facet of suicide is often overlooked: Suicide is an action. Action theory understands actions as being carried out by agents, that is by persons who are setting goals, making plans, monitoring and regulating their own behaviour, thoughts, and emotions in the pursuit of their goals [27]. The theory of goaldirected action is closely related to the concept of self-regulation [28]. Actions are related to higher-order goal-directed systems, which are shaped by a person's personality and biography. In a first case study we conceptualized suicidal behaviour using an action theoretical model [29]. We argued that suicide emerges as an alternative goal to life-oriented goals when in a person's life important life career issues or identity goals are seriously threatened.

\section{The narrative approach}

The way people make sense of the actions of others, and the way we explain our own actions is through story telling. The term narrative is defined as a story told to an attentive listener, by giving meaning to events, in order to explain the logic of a specific action. It

Citation: Konrad Michel. “Suicide Risk Assessment Must be Collaborative”. Acta Scientific Medical Sciences 4.12 (2020): 38-43. 
is also the prototype of a shared experience, or joint project. A narrative allows the patient to generate alternative perspectives and $a$ new outcome to the story. In a clinical study based on single interviews with suicide attempters we [30] found that patients' ratings of the quality of the therapeutic relationship in the Penn Helping Alliance Questionnaire HAQ [31] were significantly better when, in the opening sentence of the interview, the interviewer used a narrative approach, that is, used the words "tell" (e.g. "can you please tell me how you came to the point of harming yourself?") or "story" (e.g. "I would like to hear the story behind the suicidal crisis"). A second finding was that HAQ scores were higher when in the interview transcripts we found that interviewers acknowledged the importance of biographical issues related to suicidality ("You know, my fear of losing a close person goes right back to my childhood").

In this study, and in our clinical experience, most self-narratives had a duration of 20-30 minutes. They often start with the triggering event, then talk about past suicidal crises (often in childhood), and then move on to biographical aspects related to the suicidal behaviour, such as adverse experiences early in life (e.g. traumatic separation of parents in childhood, violence in the family, physical and sexual abuse, etc.). A truly narrative approach requires newly defined roles of the patient and the therapist: In the narrative the patient is the "expert" of his or her suicide story, the therapist being in a "not knowing position“, while in the psychiatric assessment of the patient's mental state the therapist is the expert. The Guidelines for Clinicians formulated by the Aeschi Working Group [32] emphasize that "the ultimate goal should be to engage the patient in a therapeutic relationship, even in a first assessment interview." In this concept, the personal experience and background of every single suicidal person has highest priority, not the medical diagnosis. This approach to the suicidal individual renders the existential crisis meaningful to the person in crisis, to their families, as well as to the therapists.

The potential of this new concept has been demonstrated in the scientific evaluation of the ultra-brief (three sessions) therapy program ASSIP (Attempted Suicide Short Intervention Program), which is fully based on the narrative, person-centred approach. The clinical trial included 120 patients with a recent history of attempted suicide, and compared ASSIP treatment with a control condition [33]. A survival analysis yielded an $80 \%$ risk reduction of suicide reattempts over 24 months for ASSIP-treated patients. In the ASSIP-treated group, 5 suicide reattempts were recorded, com- pared to 41 reattempts in the control group. A study carried out by the London School of Economics found that ASSIP was highly costeffective [34]. For details on the manual-based brief three-session therapy see www.assip.ch.

\section{The answer: Collaborative risk assessment}

In contrast to a traditional medical model of understanding suicide, exploring a patient's individual background of the suicidal behaviour requires a patient who is an active participant in the clinical interview. However, this also requires a clinician who can invite and activate patients to collaboratively explore the patient's individual background of the suicidal crisis, and the triggering event. The clinician needs to learn that suicidal patients have an impressive narrative competence - when the clinician offers himself as an interested, attentive listener [35]. Narratives are indispensable for the creation of meaningful and effective treatment interventions and safety planning [36]. There is only one way to deal with the problem of acute risk assessment: Join the person and make him or her an active participant in risk assessment. Teismann and colleagues [37] rightly argue that it is not the clinician who has to decide on the basis of an alleged risk categorization, but patient and therapist together need to collaboratively explore the actual suicide risk, and what the patient needs to be safe after the interview and in the following days.

Collaborative risk assessment can be done in a structured way, for instance with the Suicide Status Form SSF [38]. Here, in close collaboration with the clinician, the patient rates him/herself on a Likert scale from 1-5 on suicidal markers for psychological pain, stress, agitation, hopelessness, and self-hate in addition to making a subjective assessment of suicide risk. The patient's view is the absolute "gold standard" for risk assessment. The therapist serves as a consultant, coach and co-author.

The type of questions used to explore for suicide risk may be a barrier to collaborative risk assessment [39]. Studying video-recorded exchanges between providers and outpatients, McCabe., et al. [40] found that $75 \%$ of questions were negatively phrased, for example, "No thoughts of harming yourself?", and only $25 \%$ were positively phrased such that an expectation was set for disclosure. Questions were often close-ended and designed to elicit a yes/no answer, thus constraining any patient desire to tell their story, and to focus on their inner suffering, and explain why suicide appeared as a solution. 
However, it would be wrong to assume that a true collaboration is possible with all cases. In clinical practice the clinician's emotional responses ("gut feelings") to the patient may be indicators of risk [41]. For instance, feeling uneasy, missing the sense of collaboration and of a therapeutic relationship may be warning signs that a clinician is faced with a patient at risk. After an inpatient suicide, the clinical staff often report that they had felt uneasy with the patient, although the patient had repeatedly denied suicidal thoughts or plans.

\section{The dual role of the clinician}

The health professional in a busy ED faced with an 18-year old girl admitted after an overdose of a pain-killer will probably perceive her as withdrawn, refusing to talk about the reasons for her overdose, and will probably interpret her overdose as a way of gaining attention. However, in a collaborative approach the clinician will sit down with this girl (no parent or boyfriend in the room), try to see her as a desperate, deeply hurt human being, and ask her: "Let me try to understand, I would like you to tell me how you got to the point that you wanted to end to your life, I have got time, I left my bleep with the nurse outside". Most probably, the patient will talk about her inner pain, which had been unbearable, after her boyfriend had left her, and she will probably, without further prompting, talk about her childhood when her parents split up. However, after having established a joint understanding, the clinician needs to switch roles. To come to a comprehensive clinical assessment, the clinician will need to do a mental state exam, ask about signs and symptoms in recent days and weeks, especially signs of an affective disorder, ask about medical and psychiatric history, past suicide attempts and suicide thoughts, repeated selfharm, substance abuse, etc. and the actual psychosocial situation. A preliminary case formulation will then include a "storied description" of the suicidal crisis, the method and medical seriousness of the self-harm action, the major suicide risk factors (prior suicide attempts, suicides in the family, etc.), and a summary of the mental state exam, with a preliminary psychiatric diagnosis. Based on the above information, the clinician will then together with the patient, collaboratively develop the further procedure procedure, which may include anything from an outpatient appointment admission to inpatient care.

\section{Conclusion}

Collaborative risk assessment requires openness towards the subjective experience of the suicidal patient. In a narrative-based interview, interviewers leave behind the usual clinician's role, attributing the outsider's interpretation to a patient's suicidal be- haviour. In this clinical approach the clinician shows a genuine and empathic interest in the patient's story. This leads to a joint understanding of the suicidal crisis, and to collaboratively develop plans for the patients' needs to be safe in a short-term as well as in a long-term perspective. Risk assessment is not the clinician's own responsibility. It requires the patient's active cooperation.

\section{Bibliography}

1. Isometsä ET., et al. "The last appointment before suicide: is suicide intent communicated?" American Journal of Psychiatry 152.6 (1995): 919-922.

2. Apter A., et al. "Relationship between self-disclosure and serious suicidal behavior". Comprehensive Psychiatry 42.1 (2001): 70-75.

3. Busch KA., et al. "Clinical correlates of inpatient suicide". Journal of Clinical Psychiatry 64.1 (2003): 14-19.

4. Ajdacic-Gross V., et al. "In-patient suicide--a 13-year assessment”. Acta Psychiatrica Scandinavica 120.1 (2009): 71-75.

5. Hunt IM., et al. "Suicide in recently discharged psychiatric patients: a case-control study". Psychological Medicine 39.3 (2009): 443-449.

6. Ward-Ciesielski EF and CR Wilks. "Conducting Research with Individuals at Risk for Suicide: Protocol for Assessment and Risk Management". Suicide and Life-Threatening Behavior 50.2 (2020): 461-471.

7. Jenkins GR., et al. "Suicide rate 22 years after parasuicide: cohort study". BMJ 325.7373 (2002): 1155.

8. Owens D., et al. "Fatal and non-fatal repetition of self-harm Systematic review". The British Journal of Psychiatry 181.3 (2002): 193-199.

9. Beautrais AL. "Further suicidal behavior among medically serious suicide attempters". Suicide and Life-Threatening Behavior 34.1 (2004): 1-11.

10. Goldstein RB., et al. "The prediction of suicide. Sensitivity, specificity, and predictive value of a multivariate model applied to suicide among 1906 patients with affective disorders". Archives of Genitic Psychiatry 48.5 (1991): 418-422.

11. Posner K., et al. "The Columbia-Suicide Severity Rating Scale: initial validity and internal consistency findings from three multisite studies with adolescents and adults". American Journal of Psychiatry 168.12 (2011): 1266-1277.

12. Large M., et al. "Meta-Analysis of Longitudinal Cohort Studies of Suicide Risk Assessment among Psychiatric Patients: Heterogeneity in Results and Lack of Improvement over Time". PLoS One 11.6 (2016): e0156322. 
13. Carter G., et al. "Predicting suicidal behaviours using clinical instruments: systematic review and meta-analysis of positive predictive values for risk scales". British Journal of Psychiatry 210.6 (2017): 387-395.

14. Chan MK., et al. "Predicting suicide following self-harm: systematic review of risk factors and risk scales". British Journal of Psychiatry 209.4 (2016): 277-283.

15. Pirkis J and P Burgess. "Suicide and recency of health care contacts. A systematic review". British Journal of Psychiatry 173 (1998): 462-74.

16. Wolk-Wasserman D. "Contacts of suicidal neurotic and prepsychotic/psychotic patients and their significant others with public care institutions before the suicide attempt". Acta Psychiatrica Scandinavica 75.4 (1987): 358-372.

17. Hawton K and E Blackstock. "General practice aspects of selfpoisoning and self-injury". Psychological Medicine 6 (1976): 571-575.

18. Treolar AJ and TJ Pinfold. "Deliberate self-harm: An assessment of patients' attitudes to the care they receive". Crisis 14 (1993): 83-89.

19. Robins, E., et al. "Suicide and Attempted Suicide. England: Penguin Books Ltd. 1967. Pp. 135. \$1.25”. American Journal of Clinical Hypnosis 13.4 (1971): 289-290.

20. Harris EC and B Barraclough. "Suicide as an outcome for mental disorders. A meta-analysis". British Journal of Psychiatry 170.3 (1997): 205-228.

21. Borges G., et al. "Twelve-month prevalence of and risk factors for suicide attempts in the World Health Organization World Mental Health Surveys". Journal of Clinical Psychiatry 71.12 (2010): 1617-1628.

22. Bertolote JM., et al. "Suicide and mental disorders: do we know enough?" British Journal of Psychiatry 183 (2003): 382-383.

23. De Leo D. "Why are we not getting any closer to preventing suicide?" British Journal of Psychiatry 181 (2002): 372-374.

24. Van Praag HM. "A stubborn behaviour: the failure of antidepressants to reduce suicide rates". World Journal of Biological Psychiatry 4.4 (2003): 184-191.

25. Maltsberger JT. "The descent into suicide". The International Journal of Psychoanalysis 85.4 (2004): 653-668.

26. Tornblom AW., et al. "Shame behind the masks: the parents' perspective on their sons' suicide". Archives of Suicide Research 17.3 (2013): 242-261.
27. Gollwitzer PM. "The volitional benefits of planning, in The psychology of action. Linking cognition and motivation to behavior, P.M. Gollwitzer and J.A. Bargh, Editors. The Guilford Press: New York (1996).

28. Carver CS and M Scheier. "Principles of self-regulation: Action and emotion, in Handbook of motivation and cognition: Foundations of social behavior". E.T. Higgins and R.M. Sorrentino, Editors. 1990, Guilford Press: New York (1990): 3-52.

29. Michel K and L Valach. "Suicide as goal-directed action". Archives of Suicide Research 3.3 (1997): 213-221.

30. Michel K., et al. "Therapist sensitivity towards emotional lifecareer issues and the working alliance with suicide attempters". Archives of Suicide Research 8.3 (2004): 203-213.

31. Alexander., et al. The Penn Helping Alliance Scales, in The Psychotherapeutic Process: A Research Handbook. (1986): 325-366.

32. Michel K., et al. "Discovering the truth in attempted suicide". American Journal of Psychotherapy 56.3 (2002): 424-437.

33. Gysin-Maillart, A., et al. "A Novel Brief Therapy for Patients Who Attempt Suicide: A 24-months Follow-Up Randomized Controlled Study of the Attempted Suicide Short Intervention Program (ASSIP)". PLoS Medicine 13.3 (2016): e1001968.

34. Park AL., et al. "Cost-effectiveness of a Brief Structured Intervention Program Aimed at Preventing Repeat Suicide Attempts Among Those Who Previously Attempted Suicide: A Secondary Analysis of the ASSIP Randomized Clinical Trial". JAMA Netw Open 1.6 (2018): e183680.

35. Michel K and L Valach. "The Narrative Interview with the Suicidal Patient". in Building a Therapeutic Alliance with the Suicidal Patient, K. Michel and D.A. Jobes, Editors. American Psychological Association APA Books: Washington, DC. (2011): 63-80.

36. Warren MB and LA Smithkors. "Suicide Prevention in the U.S. Department of Veterans Affairs: Using the Evidence Without Losing the Narrative". Psychiatric Service 71.4 (2020): 398400.

37. Teismann T and W Dorrmann. "Suizidalität". Psychotherapeut 58.3 (2013): 297-311.

38. Jobes DA. "The Collaborative Assessment and Management of Suicidality (CAMS): An Evolving Evidence-Based Clinical Approach to Suicidal Risk". Suicide and Life-Threatening Behavior 42.6 (2012): 640-653.

39. Vannoy SD and LS Robins. "Suicide-related discussions with 
depressed primary care patients in the USA: gender and quality gaps. A mixed methods analysis". BMJ Open 1.2 (2011): e000198.

40. McCabe R., et al. "How do healthcare professionals interview patients to assess suicide risk?" BMC Psychiatry 17.1 (2017): 122.

\section{Assets from publication with us}

- Prompt Acknowledgement after receiving the article

- Thorough Double blinded peer review

- Rapid Publication

- Issue of Publication Certificate

- High visibility of your Published work

Website: www.actascientific.com/

Submit Article: www.actascientific.com/submission.php

Email us: editor@actascientific.com

Contact us: +919182824667 